\title{
Pachyonychia congenita
}

INSERM

\section{Source}

INSERM. (1999). Orphanet: an online rare disease and orphan drug data base.

Pachyonychia congenita. ORPHA:2309

Pachyonychia congenita $(\mathrm{PC})$ is a rare genodermatosis predominantly featuring painful palmoplantar keratoderma, thickened nails, cysts and whitish oral mucosa. 